\title{
HYDROXYAPATITE GROWTH INDUCED BY NATIVE EXTRACELLULAR MATRIX DEPOSITION ON SOLID SURFACES
}

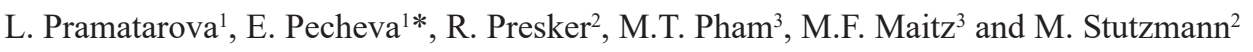 \\ ${ }^{1}$ Institute of Solid State Physics, Bulgarian Academy of Sciences, Sofia, Bulgaria \\ ${ }^{2}$ Walter Schottky Institute, Technical University, Muenchen, Germany \\ ${ }^{3}$ Institute of Ion Beam Physics and Materials Research, FZR, Dresden, Germany
}

\begin{abstract}
Biological systems have a remarkable capability to produce perfect fine structures such as seashells, pearls, bones, teeth and corals. These structures are composites of interacting inorganic (calcium phosphate or carbonate minerals) and organic counterparts. It is difficult to say with certainty which part has the primary role. For example, the growth of molluscan shell crystals is thought to be initiated from a solution by the extracellular organic matrix (ECM). According to this theory, the matrix induces nucleation of calcium containing crystals. Recently, an alternative theory has been put forward, stating that a class of granulocytic hemocytes would be directly involved in shell crystal production in oysters.

In the work presented here the surface of AISI 316 stainless steel was modified by deposition of ECM proteins. The ability of the modified substrates to induce nucleation and growth of hydroxyapatite (HA) from simulated body fluid (SBF) was examined by a kinetic study using two methods: (1) a simple soaking process in SBF and (2) a laser-liquid-solid interaction (LLSI) process which allows interaction between a scanning laser beam and a solid substrate immersed in SBF. The deposited HA layers were investigated by Fourier transform infrared spectroscopy (FTIR) and scanning electron microscopy (SEM). It was found that a coating of stainless steel surface with native ECM proteins induced nucleation and growth of HA and facilitated its crystallization. By the process of simple soaking of the samples, irrespective of their horizontal or vertical position in the solution, HA layers were grown due to the reactive ECM-coated stainless steel surface. It was shown that the process occurring in the first stages of the growth was not only a result of the force of gravity. The application of the LLSI process strongly influenced HA formation on the ECM-modified substrates by promoting and enhancing the HA nucleation and growth through a synergistic effect of a few stimuli, i.e., the modified solid surface, the laser beam and the aqueous solution.
\end{abstract}

Key words: Hydroxyapatite, native extracellular matrix, stainless steel, organic-mineral interaction, synergistic effect.

*Address for correspondence:

E. Pecheva

72 Tzarigradsko Chaussee blvd, 1784 Sofia, Bulgaria,

Telephone Number: +35927144265 ,

FAX Number: +359 $297 \underline{5} 3632$

E-mail: emily@issp.bas.bg

\section{Introduction}

The mineralization of biological tissues is a well-regulated process, which often occurs within a matrix of organic molecules. These molecules may either completely envelope or themselves be enveloped by the mineral crystals (Addadi and Weiner, 1985). They are essential for the initial deposition of mineral crystals, because they may serve as seeds for crystallization and possibly influence the number of nucleation sites generated, and also for their subsequent growth, orientation and organization (Lowenstam and Weiner, 1989). These macromolecules contribute to the strength of, and stabilize the mineral tissue. Proteins can also enhance cell adhesion and thus modulate the cellular interactions that are so important in hard tissue regeneration. Recently, an alternative theory has been put forward, stating that a class of granulocytic hemocytes would be directly involved in shell crystal production in oysters (Mount et al., 2004).

The objective of the present work was to study the ability of extracellular matrix (ECM) coated stainless steel substrates to induce hydroxyapatite (HA) growth from simulated body fluid (SBF). To understand the mechanism of the HA formation, a kinetic study by two methods was performed: (1) a simple soaking process in SBF and (2) a laser-liquid-solid interaction (LLSI) process. Direct nucleation of HA on ECM proteins was performed to prepare a 'steel-ECM-HA' composite as a new attractive model system for studying the principles of biomineralization. Stainless steel has been applied for many years in orthopaedics and dentistry for hard tissue implants. Lasers have been widely used as a source of energy in the area of biomaterials and have found applications in pulsed laser deposition and processing of the HA, in biofilm modification, bone defect healing and modification of root canal dentine (Cotell, 1993; Richter et al., 2001; Guzzardella et al., 2002; Ebihara et al., 2002). However, to the best of our knowledge, laser irradiation of a solid substrate immersed in aqueous solution has not been used in the process of HA deposition with a view to enhancing this process. In this experiment, a few stimuli were applied simultaneously in the LLSI process to attain a synergistic effect on HA crystal growth.

\section{Materials and Methods}

Samples $(8 \times 8 \times 1 \mathrm{~mm})$ were prepared by coating of standard austenitic stainless steel AISI 316 with a native ECM (further named ECM/SS). The osteoblast-like cell line SAOS-2 was allowed to synthesize and assemble its own ECM on the substrates under standard cell culture 


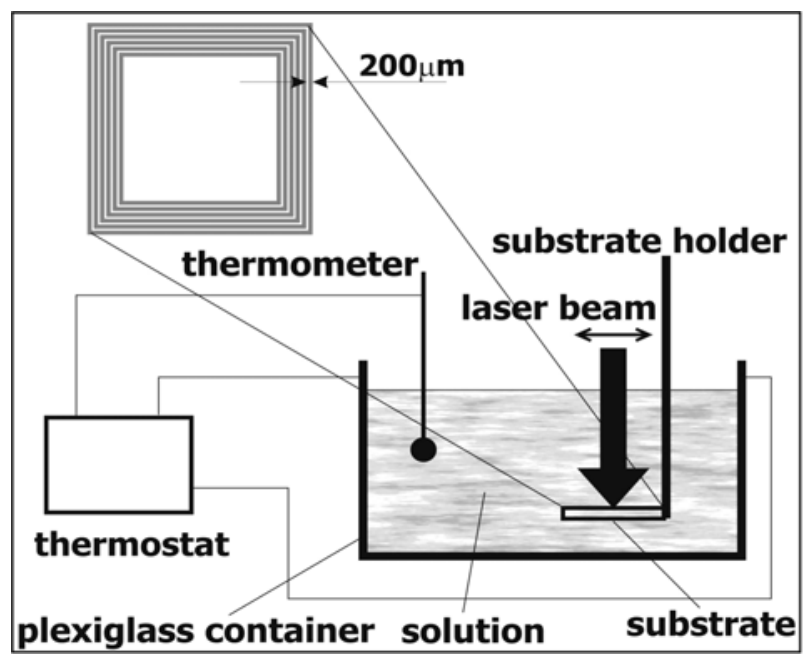

Figure 1. Schematic of the experimental set-up. In the upper left corner, the design of the laser irradiation in the LLSI process is shown.

conditions (Pham et al., 2003). Cells were then selectively removed, which resulted in surfaces coated with a thin film of ECM. To evaluate the ability of the modified samples to induce HA growth, a kinetic study by two methods was performed. In the first method, a simple soaking process in SBF was carried out (described in Pecheva et al., 2004a) for a duration of 4 and $24 \mathrm{~h}$, and the samples were immersed horizontally or vertically in the solution (in the following these samples are named ECM/SS_hs and ECM/SS_vs, respectively). For the preparation of the fluid, reagent-grade chemicals $\left(\mathrm{NaCl}, \mathrm{NaHCO}_{3}, \mathrm{KCl}, \mathrm{K}_{2} \mathrm{HPO}_{4}-3 \mathrm{H}_{2} \mathrm{O}, \mathrm{MgCl}_{2}-\right.$ $\left.6 \mathrm{H}_{2} \mathrm{O}, \mathrm{Na}_{2} \mathrm{SO}_{4}-10 \mathrm{H}_{2} \mathrm{O}, \mathrm{CaCl}_{2}-6 \mathrm{H}_{2} \mathrm{O}\right)$ were dissolved in distilled water and buffered at $\mathrm{pH} 7.4$ with trishydroxymethyl-aminomethane buffer or hydrochloric acid. The $\mathrm{Ca}$ and $\mathrm{P}$ concentrations were 1.5 times higher than the ones used by Kokubo (Kokubo et al., 1990). In the second method, a novel process named LLSI was applied. This process involves interaction between a scanning laser beam and the modified substrate immersed in SBF, followed by soaking in the same SBF in a horizontal position for 4 and $24 \mathrm{~h}$ (named ECM/SS LLSI $4 \mathrm{~h}$ and ECM/SS LLSI 24h). To study the instantaneous effect of the laser energy on HA nucleation and growth some samples were taken out immediately after laser irradiation (named ECM/SS_LLSI_0h). The experimental set-up is shown in Fig. 1. It included an open deposition system, where the liquid precursor $(400 \mathrm{ml}$ SBF kept at a temperature of $37^{\circ} \mathrm{C}$ and $\mathrm{pH} 7.4$ ) was placed in a reaction container and the temperature was controlled by a thermostat. The solid substrate was immersed in the SBF and irradiated by the laser beam from a $\mathrm{CuBr}$ vapor pulsed laser ( $\lambda=578.2 \mathrm{~nm}, 330 \mathrm{~mW}$ ) equipped with a scanning system. The laser beam was directed perpendicularly to and focused on the substrate surface, and the interaction time was within 5 min. By scanning the surface, a design of seven squares at a distance of $200 \mu \mathrm{m}$ was created at the end of each sample (Fig. 1, upper left corner). In this way the center of the substrate was not irradiated. The morphology of the deposited HA layers was investigated by scanning electron microscopy (SEM, DSM 962, Zeiss,

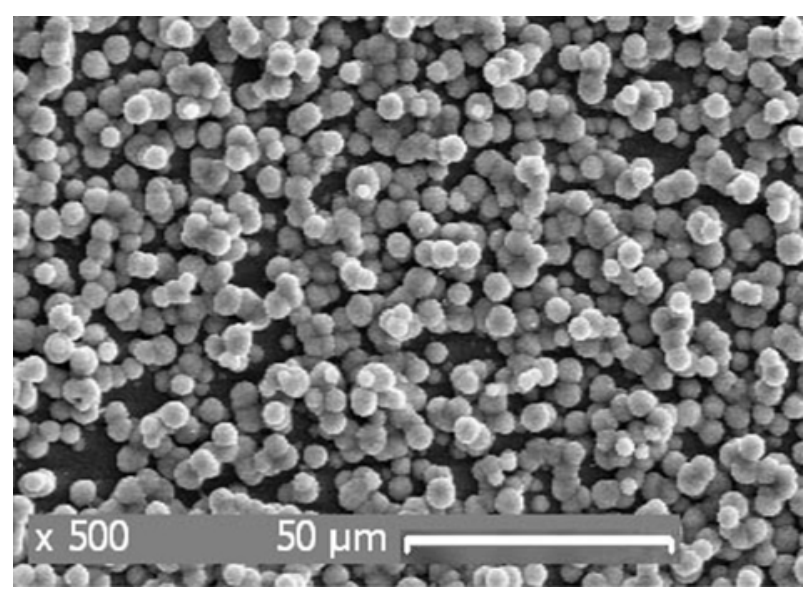

Figure 2. The morphology of the HA layer grown on ECM/SS, observed by SEM

Oberkochen, Germany). The relative thickness of HA layers was measured by Fourier transform infrared (FTIR) spectroscopy (IRscope II, Bruker Optics IFS 66/S, Germany, reflection mode).

\section{Results}

Figure 2 demonstrates the morphology of the grown HA layers obtained by a simple soaking process for a period of over $24 \mathrm{~h}$. The SEM image shows regular spherical aggregates with an average size of $5 \mu \mathrm{m}$, grouped homogeneously in a network and embedded in the ECM surface as in a matrix.

FTIR spectra, recorded at different intervals of time are shown in Fig. 3. The shown spectra in Fig 3a were recorded after a simple soaking process (horizontal and vertical positions of the samples in the SBF) for a period of 4 and 24 h. In Fig. 3b, the FTIR spectra of the grown HA layer by applying a LLSI process are shown. These spectra were recorded immediately after laser irradiation, as well as after subsequent soaking for periods of 4 and $24 \mathrm{~h}$. FTIR reflection spectra measured in the region 800 $\mathrm{cm}^{-1}-4000 \mathrm{~cm}^{-1}$ showed typical vibration modes of HA on the stainless steel surfaces modified by the two methods. The $v_{1}$ and $v_{3}$ P-O stretching vibrations were registered at wavenumbers in the region of $962-1113 \mathrm{~cm}^{-1}$. The incorporation of carbonate was deduced from the shoulder at $835-876 \mathrm{~cm}^{-1}$, characteristic for $\mathrm{v}_{2} \mathrm{C}-\mathrm{O}$ asymmetric bending in $\mathrm{CO}_{3}{ }^{2-}$. A band at $1650 \mathrm{~cm}^{-1}$ and a broad band from $2500-3700 \mathrm{~cm}^{-1}$ in the spectra contributed to the $\mathrm{H}$ $\mathrm{O}-\mathrm{H}$ vibrations in adsorbed $\mathrm{H}_{2} \mathrm{O}$. It was seen from the spectra that soaking of the vertically oriented samples induced a thin HA layer on their surfaces. The FTIR reflection from the HA layers grown by the two methods showed a different peak intensity. An estimate of the relative layer thickness using the Buger-Lambert-Beer's law for the optical density at $962 \mathrm{~cm}^{-1}$ (Fig. 4) was performed according to Pecheva et al. (2004a). The thinnest layers were observed on samples, soaked vertically for 4 and $24 \mathrm{~h}$, and samples soaked horizontally for $4 \mathrm{~h}$. The calculated optical density (in arbitrary units) was 0.05 for SS/ECM_vs_4h and SS/ECM_vs_24h, and 

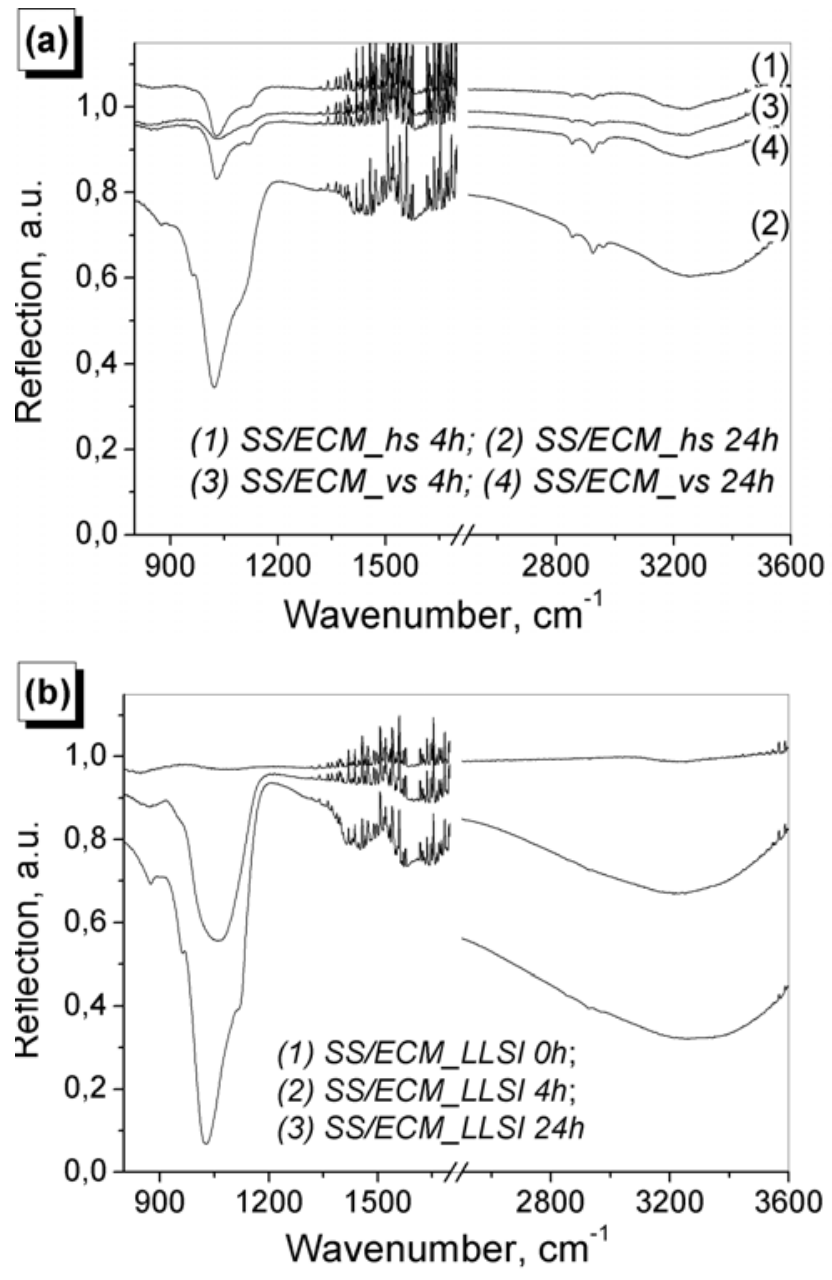

Figure 3. FTIR spectra of the HA layer grown on ECM/ SS by: (a) a simple soaking in SBF for $4 \mathrm{~h}$ and $24 \mathrm{~h}$; (b) a laser-liquid-solid interaction in SBF and subsequent soaking for $0 \mathrm{~h}, 4 \mathrm{~h}$ and $24 \mathrm{~h}$.

0.08 for SS/ECM_hs_4h. When a horizontal soaking for 24 $\mathrm{h}$ was applied (SS/ECM_hs_24h) the optical density increased to 0.50 . In comparison to the simple soaking, the employment of the LLSI process yielded a higher optical density: 0.18 for $4 \mathrm{~h}$ of subsequent soaking (SS/ ECM_LLSI_4h) and 0.64 for the samples soaked for $24 \mathrm{~h}$ (SS/ECM_LLSI_24h). The HA bands in the FTIR spectra of the samples taken out immediately after the laser irradiation were hardly discernible, while after $4 \mathrm{~h}$ they were clearly observed and showed a low layer perfection. With increasing time of soaking ( $24 \mathrm{~h}$ soaking) after the LLSI process, a higher degree of crystallinity of the HA layer was observed (Fig. 3 b).

\section{Discussion}

It was observed that a stainless steel surface, coated by ECM, induced a layer consisting of regular spherical aggregates with an average size of $5 \mu \mathrm{m}$, grouped homogeneously in a network. The SEM image showed that these aggregates were embedded in the ECM surface as in a matrix, a property that was ascribed to the ability of the organic molecules to control and define the crystallization process by serving as a template for the mineralization, a fact observed by many researchers
(Addadi and Weiner, 1989; Lowenstam and Weiner, 1989; Aizenberg , 2000; Mann et al., 1993). In our previous experiments (Pecheva et al., 2004b; Pecheva et al., 2004c; Pramatarova et al., 2004), when a layer had been grown on $\mathrm{Ca}$ and $\mathrm{P}$ ion-implanted or on nonmodified surfaces, the aggregates had an irregular spherelike shape and smaller sizes (1-2 $\mu \mathrm{m})$ and were spread inhomogeneously on the sample surface, forming highly porous layers. The long-distance order of the aggregates was detected only in the case of ECM-modified stainless steel.

The main phase detected with FTIR spectroscopy after the simple soaking process and after LLSI was HA. In addition, we assumed that an acid calcium phosphate phase, octacalcium phosphate, was also present in small amounts in the HA layers but since its wavenumber coincided with the $\mathrm{v}_{2} \mathrm{C}-\mathrm{O}$ bending (the $\mathrm{P}-\mathrm{OH}$ stretching mode in $\mathrm{HPO}_{4}{ }^{2-}$ is usually observed at around $870 \mathrm{~cm}^{-1}$ ) this could not be easily proven. In our experiment, the measured FTIR spectra were in accordance with the data in the literature for the biological calcium phosphates (LeGeros and LeGeros, 1984). According to these data, carbonate groups are present in the spectra of biological calcium phosphates and are considered to be a principal cause of distortion in the structure of most biological apatites. Water, observed in our spectra by the bands at $1650 \mathrm{~cm}^{-1}$ and from $2500-3700 \mathrm{~cm}^{-1}$ is present in human tooth enamel and biological apatites (LeGeros and LeGeros, 1984). Thus, the $\mathrm{CO}_{3}$-containing HA layers induced by ECM deposition resembled the biological HA. Keeping in mind the morphology of the aggregates and their order, observed by SEM, it could be concluded that the ECM proteins facilitated the mineralization on the stainless steel surface by serving as an in-vitro matrix for HA growth and contributing to its crystallization. This result could be successfully used in both studying the mechanism of the mineralization process in nature and in tissue engineering.

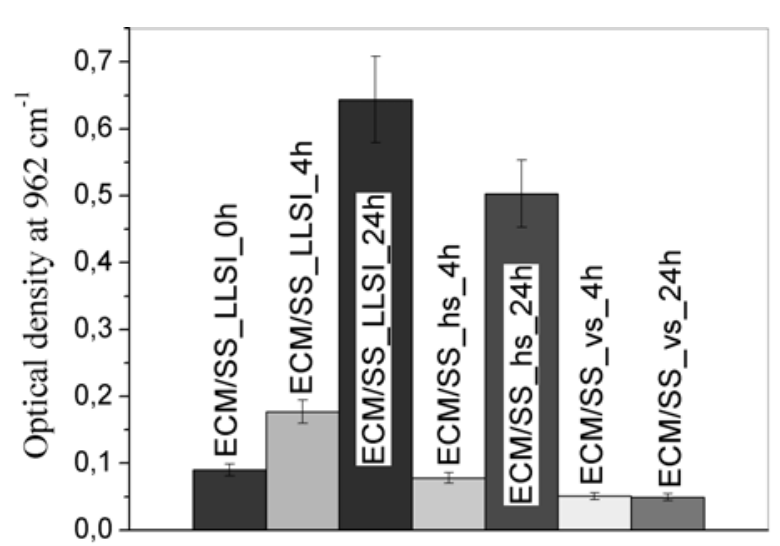

Figure 4. Kinetic diagram of the calculated optical density at the $962 \mathrm{~cm}^{-1}$ band, derived from FTIR spectra of the samples, obtained by simple soaking process in the solution; for samples placed horizontally (hs) and vertically (vs) for $4 \mathrm{~h}$ and $24 \mathrm{~h}$ ( $\mathrm{rms} \pm 0.01$ ), as well as for the samples obtained for $0 \mathrm{~h}, 4 \mathrm{~h}$, and $24 \mathrm{~h}$ by applying the LLSI process $(\mathrm{rms} \pm 0.02)$. 
Our previous X-ray diffraction investigation (Pecheva et al., 2004c) of the layers grown on ECM-modified and non-modified stainless steel substrates indicated that the HA layer grown in the presence of surface ECM proteins was thicker and with a higher crystallinity. A calculation by using the isolated (002) reflection of the HA gave a value for the grain size of $46 \mathrm{~nm}$, compared to $34 \mathrm{~nm}$ for the grain size of the HA grown on non-modified surfaces.

To examine the mechanism of crystal growth, the samples were positioned horizontally or vertically in the solution. Optical densities determined from FTIR spectra (Fig. 4) showed that the samples soaked for $4 \mathrm{~h}$ in the vertical position had a HA layer on their surfaces with a thickness comparable to that of samples soaked for the same period in the horizontal position, which confirmed that the layer deposition was not simply a question of gravity. Further soaking for $24 \mathrm{~h}$ did not increase the thickness of the layer existing after $4 \mathrm{~h}$, i.e., the reactivity of the ECM-modified surfaces significantly decreased and the velocity of HA growth was very slow (Fig. 4).

The thicker layer on the horizontally placed samples after $24 \mathrm{~h}$ of soaking in SBF was attributed to precipitation in combination with the action of the force of gravity. When the LLSI process was applied, the formation of a very thin layer was observed in the spectra immediately after the samples have been taken out of the SBF (SS/ ECM_LLSI_0h), i.e., this step was regarded as a very fast (within a few minutes) formation of nuclei, which was not feasible by the simple soaking. Furthermore, after soaking of the laser irradiated samples for 4 and $24 \mathrm{~h}$, a thick HA layer have been grown facilitated by the already formed nuclei. This layer was 2.3 and 1.3 times thicker, respectively, than the layers formed in the soaked samples without LLSI. Peak fitting was applied to the spectra of the 4 and 24 h-soaked samples. The full width half maximum (FWHM) of the peak at $1120.6 \mathrm{~cm}^{-1}$ characteristic of poorly crystalline HA decreased from 63.3 $\mathrm{cm}^{-1}$ to $43.4 \mathrm{~cm}^{-1}$, i.e. the contribution of crystalline material increased. It was concluded that the applied laser energy strongly influenced HA formation on the substrates modified by ECM by promoting and enhancing the HA nucleation and growth. Probably, the few stimuli applied simultaneously in the LLSI process, i.e., the laser beam, the solid surface and the aqueous solution had a synergistic effect on the HA crystal growth. This result was achieved by an easy and quick one-step process of simultaneous application of the laser energy in the SBF.

\section{Acknowledgements}

This research was supported by a Marie Curie grant of the EC (HPMT-CT-2000-00182), by the Bulgarian National Scientific Research Fund (Grant L1213) and partly by a Deutsche Forschungsgemeinschaft (DFG) grant. We thank PULSLIGHT Ltd for the laser equipment.

\section{References}

Addadi L, Weiner S (1985). Interactions between acidic proteins and crystals: Stereochemical requirements in biomineralization. Proc Natl Acad Sci USA 82: 4110-4114.

Aizenberg J (2000). Patterned crystallization of calcite in vivo and in vitro. J Crys Growth 211: 143-148.

Cotell C (1993). Pulsed laser deposition and processing of biocompatible hydroxyapatite thin films. Appl Surf Sci 69: $140-148$.

Ebihara A, Majaron B, Liaw L, Krasieva T, WilderSmith P (2002). Er:YAG laser modification of root canal dentine: influence of pulse duration, repetitive irradiation and water spray. Lasers Med Sci 17: 198-207.

Guzzardella G, Fini M, Torricelli P, Giavaresi G, Giardino R (2002). Laser stimulation on bone defect healing. An in vitro study. Lasers Med Sci 17: 216-220.

Kokubo T, Kushitani H, Sakka S, Kitsugi T, Yamamuro $\mathrm{T}$ (1990). Solutions able to reproduce in vivo surfacestructure changes in bioactive glass-ceramic A-W ${ }^{3}$. J Biomed Mater Res 24: 721-734.

LeGeros RZ, LeGeros JP (1984) Phosphate minerals in human tissues. In: Phosphate Minerals. Nriagu JO, Moore PB (eds). Springer Verlag, New York, p. 351-385.

Lowenstam H, Weiner S (1989). On Biomineralization. Oxford University Press, Oxford, pp 74-175.

Mann S, Archibald D, Didymus J, Douglas T, Heywood B, Meldrum F, Reeves N (1993). Crystallization at inorganic-organic interfaces: biominerals and biomimetic synthesis. Science 261: 1286-1292.

Mount A, Wheeler A, Paradkar R, Snider D (2004). Hemocyte-mediated shell mineralization in the eastern oyster. Science 304: 297-300.

Pecheva E, Pramatarova L, Maitz MF, Pham MT, Kondyuirin A (2004a). Kinetics of hydroxyapatite deposition on solid substrates modified by sequential dual implantation of $\mathrm{Ca}$ and $\mathrm{P}$ ions. Part I. FTIR and Raman spectroscopy study. Appl Surf Sci 235: 176-181.

Pecheva E, Pramatarova L, Maitz MF, Pham MT, Kondyuirin A (2004b). Kinetics of hydroxyapatite deposition on solid substrates modified by sequential dual implantation of $\mathrm{Ca}$ and $\mathrm{P}$ ions. Part II. Morphological, composition and structure study. Appl Surf Sci 235: 170175.

Pecheva E, Pramatarova L, Maitz MF, Pham MT, Kondyurin A (2004c). Extracellular matrix used in-vitro model system for hydroxyapatite formation. Ann Transpl 9: 58-60.

Pham MT, Reuther H, Maitz MF (2003). Native extracellular matrix coating on Ti surfaces. J Biomed Mater Res 66A: 310-316.

Pramatarova L, Pecheva E, Petrov T, Minkovski N, Kondyurin A, Pramatarova R (2004). Enhancement of hydroxyapatite formation by laser-liquid-solid interaction. Proc SPIE 5449: 46-50.

Richter A, Gonprot P, Smith R (2001). Biofilms and their modification by laser irradiation. Nucl Instrum Methods Phys Res B 180: 1-11. 